\title{
Influencia de la porosidad en las propiedades magnéticas de arreglos de nanohilos de $\mathrm{Ni}$
}

\author{
Influence of the porosity on the magnetic \\ properties of Ni nanowires arrays
}

Fernando Meneses ${ }^{1}$, Paula Gabriela Bercoff ${ }^{1,2}$

\author{
${ }^{1}$ Fa.M.A.F., Universidad Nacional de Córdoba - M. Allende s/n, Ciudad Universitaria - CP 5000 - Córdoba, Argentina \\ e-mail: fmenesesunc@hotmail.com \\ ${ }^{2}$ Instituto de Física Enrique Gaviola, CONICET - M. Allende s/n, Ciudad Universitaria - CP 5000 - Córdoba, Argentina \\ e-mail: bercoff@famaf.unc.edu.ar
}

\begin{abstract}
RESUMEN
Se estudiaron las propiedades de histéresis magnética de arreglos ordenados de nanohilos de Ni, electrodepositados éstos en una membrana de alúmina con un arreglo hexagonal de poros cilíndricos. Se analizó el efecto de la geometría de los nanohilos individuales (diámetro y longitud) y del ordenamiento y porosidad del arreglo sobre la coercitividad y la remanencia del conjunto. Las membranas de alúmina auto-ensambladas se sintetizaron por anodizado en dos pasos, usando una celda electroquímica y potencial eléctrico constante. Se variaron los diámetros de poro entre $20 \mathrm{~nm}$ y $40 \mathrm{~nm}$ (no así la distancia media entre ellos) sumergiendo las membranas en ácido fosfórico al 5\% durante distintos tiempos. Estas membranas se caracterizaron por microscopía de barrido (SEM). Los nanohilos de Ni se obtuvieron por electrodeposición AC, a 20 V, 200 Hz y temperatura ambiente. Una vez sintetizados, los arreglos de nanohilos se caracterizaron estructuralmente por SEM y magnéticamente usando un SQUID. Se observa que las propiedades magnéticas de los arreglos son fuertemente dependientes de la relación largo/diámetro de los nanohilos, la que regula el valor de su anisotropía de forma, y también de la porosidad, que determina la magnitud del campo de interacción dipolar que percibe cada nanohilo por la presencia de sus vecinos en el arreglo. En particular, se encontró que la coercitividad decrece con la porosidad y que el parámetro $\varepsilon$, que regula la intensidad de las barreras de energía del sistema, debe ser variable y depender de la porosidad del arreglo para ajustar bien los datos experimentales.
\end{abstract}

Palabras clave: Nanohilos, Propiedades magnéticas, Níquel

\section{ABSTRACT}

The hysteresis properties of ordered arrays of Ni nanowires, electrodeposited into cylindrical nanoporous alumina membranes were investigated. The effect of the nanowire geometry (diameter and length), the array order and the nanowires' volume fraction on the array coercivity and remanence were analyzed. Porous alumina membranes were produced by a two-step anodization process in a potentiostatic electrochemical cell. The pores' diameters were varied from $20 \mathrm{~nm}$ to $40 \mathrm{~nm}$ by immersing the membranes in a 5\% phosphoric acid solution for different times. The membranes were characterized using a scanning electron microscope (SEM) and both the pore size distribution as well as their separation were determined. These membranes were used as templates for the AC electrodeposition of Ni nanowires, using a voltage of $20 \mathrm{~V}$ and a frequency of $200 \mathrm{~Hz}$, at room temperature. The Ni nanowire arrays were structurally and magnetically characterized by SEM and with a SQUID magnetometer, respectively, at room temperature. It was found that the magnetic properties of the array strongly depend on the nanowires aspect ratio -length/diameter ratio- which regulates the wire shape anisotropy and also on the porosity, which determines the magnitude of the dipolar interaction field acting on the array. Particulary, it was found that coercitivity decreases with porosity and that the parameter $\varepsilon$, which is related to the energy barriers of the system, must depend on the porosity of the ensemble in order to accurately describe the experimental data.

Keywords: Nanowires, Magnetic properties, Nickel 


\section{INTRODUCCIÓN}

Los nanomateriales ofrecen la oportunidad de estudiar nuevos fenómenos y generar dispositivos novedosos para la (nano)electrónica, la catálisis, la remediación ambiental y la producción de energía (entre otras aplicaciones) debido a las propiedades especiales que se originan como consecuencia del confinamiento estructural [1-8] . En particular, resulta atractivo el estudio de sistemas nanométricos metálicos (como el Ni) debido a su pequeña anisotropía magneto-cristalina y potenciales aplicaciones en dispositivos magnéticos [ㅁ-르].

La electrodeposición de materiales dentro de los poros de membranas que presentan arreglos ordenados es una técnica exitosa para la síntesis de nanomateriales dado que dichas membranas se pueden obtener de manera simple y a bajo costo. Los factores relevantes para determinar las propiedades de estos sistemas son (además de la composición de los hilos) el diámetro, la relación longitud/diámetro y la densidad del empaquetamiento de los nanohilos en el arreglo. Asimismo, la rugosidad de la superficie, si son mono o policristalinos, y en este caso la existencia o no de textura cristalográfica, como también la orientación del eje de fácil magnetización relativa al eje longitudinal del hilo son factores determinantes.

Los nanohilos electrodepositados en los poros ordenados de una membrana de alúmina se encuentran físicamente aislados unos de otros; sin embargo, están sujetos a una interacción magnética dipolar controlable, que depende de la simetría del arreglo de poros y de la distancia media inter-poro [13]. Esta interacción dipolar condiciona, en algunos casos, la inversión de la polarización del arreglo [14]. La histéresis magnética de los nanohilos individuales está determinada por la anisotropía magnética efectiva, la que en general resulta de varias contribuciones de distinta naturaleza, como la anisotropía magneto-cristalina, la anisotropía de forma y/o la magneto-elástica. Esto da lugar a variaciones complejas de la polarización de saturación, la coercitividad y la cuadratura del lazo de histéresis.

En el caso del Ni, la anisotropía magneto-cristalina es muy baja comparada con la de otros metales cúbicos como, por ejemplo, el Fe (-5 x $10^{3} \mathrm{~J} / \mathrm{m}^{3}$ para el Ni y 4,8 x $10^{4} \mathrm{~J} / \mathrm{m}^{3}$ para el Fe [15]). Por lo tanto, en el caso de nanohilos de $\mathrm{Ni}$, se espera que la anisotropía de forma sea la preponderante. Con el objeto de estudiar la influencia de la anisotropía de forma y de la interacción dipolar sobre el comportamiento magnético de arreglos ordenados de nanohilos de $\mathrm{Ni}$, se prepararon muestras con distintas relaciones de aspecto longitud/diámetro $(A r=L / d)$ y diferentes empaquetamientos de hilos. Se midieron los lazos de histéresis magnética en configuración PA (campo aplicado paralelo al eje longitudinal del hilo) y PE (campo aplicado perpendicular a este eje).

\section{MATERIALES Y MÉTODOS}

La producción de nanohilos se llevó a cabo en dos etapas: primero se procedió a la fabricación de la membrana porosa de alúmina y luego se efectuó la electrodeposición de los iones metálicos dentro de los poros de la membrana.

La caracterización de las estructuras obtenidas se realizó por microscopía de barrido con un microscopio FE-SEM Sigma Zeiss y por magnetometría con un SQUID, a temperatura ambiente y variando el campo aplicado entre -2 y 2 T. Los arreglos de nanohilos se midieron en dos configuraciones diferentes: una aplicando el campo magnético en dirección paralela (PA) al eje mayor de los nanohilos (out of plane configuration) y la otra perpendicular (PE) a dicho eje, contenido en el plano del sustrato (in plane configuration). A continuación se describen las etapas de producción de las membranas y los nanohilos en detalle.

\subsection{Fabricación de membrana porosa}

Se usó como sustrato original una lámina de aluminio comercial de pureza 99,997\% (Puratronic®) y de 0,2 mm de espesor. La preparación comenzó con la inmersión de la lámina en un baño de acetona para desengrasar la superficie. Luego se realizó un pulido electroquímico en un baño ácido a un voltaje de $20 \mathrm{~V}$ durante 5 minutos. Para remover cualquier tipo de óxido restante se sumergió la lámina en una solución de $\mathrm{NaOH} 1 \mathrm{M}$. Una vez preparado el sustrato, se realizó el anodizado en dos pasos (primer y segundo anodizado) utilizando una celda electroquímica, con la lámina de aluminio actuando como ánodo del sistema y una barra de grafito inerte como cátodo, ambos inmersos en un baño electrolítico.

Las dos etapas del anodizado se realizaron a voltaje constante, obteniéndose membranas con un espesor aproximado de $1 \mu \mathrm{m}$. La temperatura del electrolito durante todo el proceso se mantuvo constante e igual a $2{ }^{\circ} \mathrm{C}$. Para lograr diámetros de poro de $20 \mathrm{~nm}$ se utilizó como electrolito ácido oxálico $(\mathrm{COOH})_{2}$ 0,3M [16]. Después del primer anodizado, e inmediatamente antes del segundo, se removió la capa de óxido formada (desordenada) realizando un ataque químico con una solución de ácido crómico y ácido fosfórico a $60{ }^{\circ} \mathrm{C}$. La Fig. 1(a) muestra un esquema del proceso completo de producción de la membrana y en la Fig. 1(b) puede observarse una fotografía SEM de la parte superior de la membrana luego de este proceso. Las membranas 
así obtenidas fueron sumergidas en una solución de ácido fosfórico al 5\% en volumen por 5, 10, 20 y 30 minutos para obtener poros de distintos diámetros $d$. Con estos tiempos de inmersión se consiguieron poros de diámetros $d$ entre $21 \mathrm{~nm}$ y $40 \mathrm{~nm}$. Los valores de los parámetros geométricos de los nanoporos en la membrana de alúmina se listan en la Tabla 1.

\subsection{Síntesis de nanohilos}

Los nanohilos de Ni se obtuvieron por electrodeposición en una celda electroquímica alimentada por una fuente AC, con frecuencia y voltaje de trabajo de $200 \mathrm{~Hz}$ y $20 \mathrm{~V}$, respectivamente. Actuaron como electrodos una barra de grafito de alta densidad y el sustrato de $\mathrm{Al}$ donde se formó la membrana porosa (ver Fig. 1(c)).

El electrolito utilizado consistió en una solución acuosa de sulfato de Ni y ácido bórico 0,3 M, donde el mecanismo de reducción $\mathrm{Ni}^{2+}+2 e \leftrightharpoons \mathrm{Ni}$ es favorecido por el ácido bórico, que se adsorbe débilmente en la superficie del electrodo e inhibe el desprendimiento de hidrógeno [17]. También, el ácido bórico actúa como buffer, disminuyendo el cambio de $\mathrm{pH}$ en la superficie del electrodo y evitando la formación de óxidos [17]. El pH del electrolito se mantuvo ajustado en 5 . El tiempo de electrodeposición fue de 1,5 minutos para las cuatro membranas utilizadas.

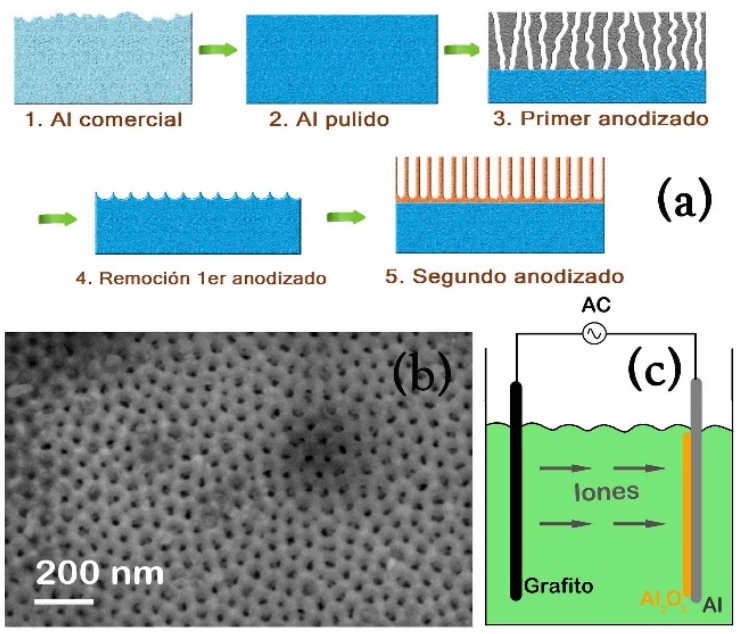

Figura 1: (a) Esquema del proceso de síntesis de membranas porosas; (b) Vista superior de una membrana luego del segundo anodizado; (c) Esquema de la celda usada para electrodeposición.

\section{RESULTADOS Y DISCUSIÓN}

En las Figs. 2 (a)-(d) se muestran imágenes de las membranas obtenidas luego del baño en ácido fosfórico por distintos tiempos, para variar los diámetros $d$ de los poros (la membrana sin sumergir se muestra en la Fig.

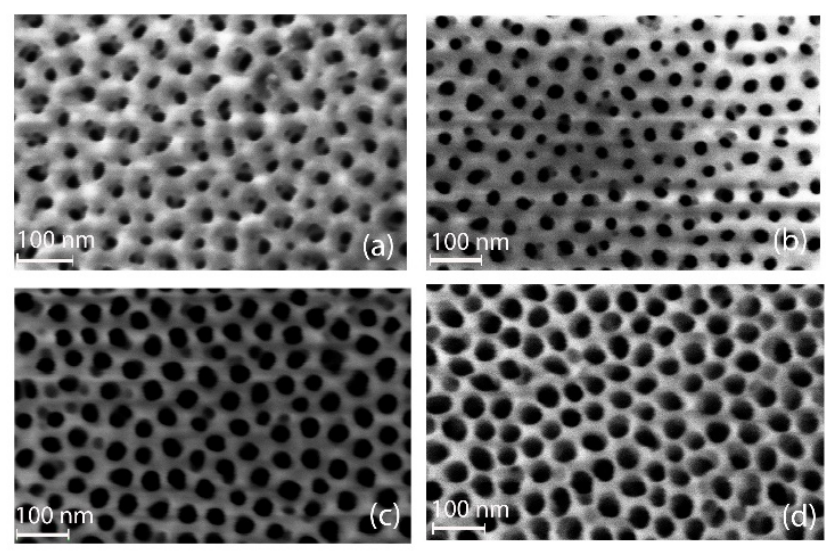

$1(\mathrm{~b}))$.

Figura 2: Vista superior de la membrana de alúmina luego de un baño en solución de ácido fosfórico al 5\% por (a) 5 minutos, (b) 10 minutos, (c) 20 minutos y (d) 30 minutos. 
El tiempo de electrodeposición elegido (1,5 minutos) no alcanzó para el llenado completo de los poros. En todos los casos se obtuvieron hilos con distribuciones de longitud $L$ que van entre los $450 \mathrm{~nm}$ (para poros con $d=(21 \pm 2) \mathrm{nm})$ y $200 \mathrm{~nm}$ (poros $d=(40 \pm 2) \mathrm{nm})$, lo cual resulta en diferentes relaciones de aspecto $\mathrm{Ar}$ (cociente entre la longitud $L$ y el diámetro $d$ : $A r=L / d$ ) para los nanohilos obtenidos. En la Fig. 3 se muestra una visión lateral de una de las muestras, representativa del resto, donde pueden observarse los nanohilos en la parte inferior de la membrana.

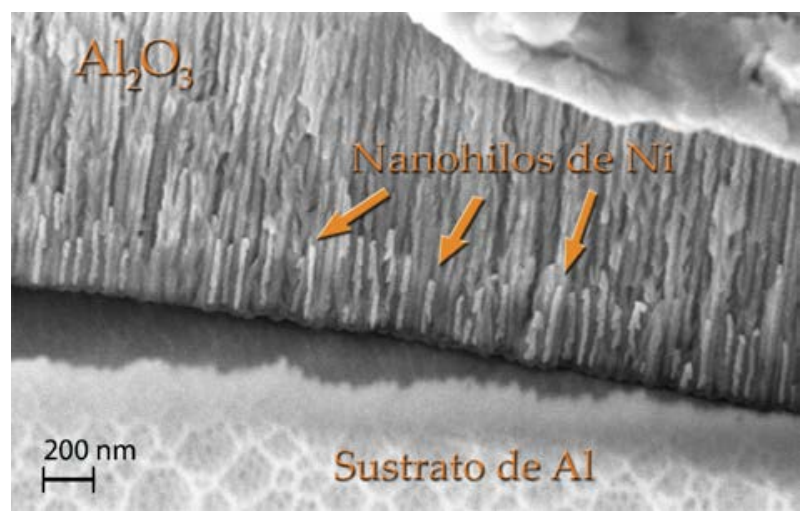

Figura 3: Imagen de los nanohilos de $\mathrm{Ni}$ en la membrana de alúmina. Los hilos pueden verse en la parte inferior, con mayor contraste debido al mayor número atómico del Ni con respecto a la alúmina. La micrografía corresponde a los hilos formados en la membrana con poros de $30 \mathrm{~nm}$, y es representativa de las demás.

En la Tabla 1 se describen los parámetros geométricos de cada muestra, que fueron determinados a partir de varias fotografías SEM, tomando un gran número de datos para reducir la incerteza. Se observa que la dispersión en el diámetro $d$ es mucho menor que la que se obtiene para las longitudes $L$ de los hilos.

Tabla 1: Denominación de las muestras estudiadas y sus correspondientes parámetros geométricos.

\begin{tabular}{c|c|c|c|c|c|c}
\hline Muestra & $\boldsymbol{d}[\mathrm{nm}]$ & $\boldsymbol{L}[\mathrm{nm}]$ & $\boldsymbol{A r}$ & $\begin{array}{c}\text { Espesor de la pared de la } \\
\text { membrana [nm] }\end{array}$ & $\boldsymbol{P}$ (medida) & $\boldsymbol{P}$ (calculada) \\
\hline $\mathrm{Ni}-21$ & $21 \pm 2$ & $450 \pm 30$ & $21 \pm 2$ & $34 \pm 8$ & $0,12 \pm 0,01$ & $0,13 \pm 0,02$ \\
\hline $\mathrm{Ni}-30$ & $30 \pm 2$ & $350 \pm 30$ & $12 \pm 2$ & $25 \pm 8$ & $0,25 \pm 0,02$ & $0,26 \pm 0,02$ \\
\hline $\mathrm{Ni}-33$ & $33 \pm 2$ & $200 \pm 30$ & $6 \pm 1$ & $22 \pm 8$ & $0,30 \pm 0,01$ & $0,31 \pm 0,02$ \\
\hline $\mathrm{Ni}-40$ & $40 \pm 2$ & $200 \pm 30$ & $5 \pm 1$ & $15 \pm 8$ & $0,41 \pm 0,02$ & $0,46 \pm 0,02$ \\
\hline
\end{tabular}

Debido al método de obtención de membranas con distintos diámetros $d$ de poro que se utilizó (por inmersión en ácido fosfórico por distintos tiempos), la distancia interporo $D$, definida como la longitud entre centro y centro de dos poros vecinos (que queda determinada por el voltaje de anodizado), es la misma para todas las muestras: $D=(56 \pm 6) \mathrm{nm}$. De esta manera, el espesor de la pared interporo disminuye con el tiempo de inmersión, como puede constatarse en la Tabla 1. Una variable que tiene en cuenta estos parámetros es la porosidad de la membrana $P$ (o densidad de empaquetamiento de los poros), que se define como:

$$
\begin{aligned}
& P=\left(\frac{n^{\circ} \text { de poros }}{\text { área }}\right)(\text { área transversal de un poro }) \\
& P=\frac{\pi}{2 \sqrt{3}}\left(\frac{d}{D}\right)^{2}
\end{aligned}
$$

Para las muestras estudiadas, se midió $P$ usando la primera definición dada por la ec. (1) utilizando varias imágenes SEM y se la calculó empleando la segunda definición (ec. (2)), que tiene en cuenta el empaquetamiento hexagonal compacto ideal en el plano. Ambos resultados se listan en la Tabla 1. El acuerdo entre los valores es notable, indicando el alto grado de orden hexagonal obtenido en las membranas. Por otra parte, K. NIELSCH et al. [18] encontraron que para membranas de alúmina con un autoensamblado perfecto (es decir con el mayor ordenamiento posible), la porosidad ideal debe ser del 10\%, que es el valor que se obtiene para la membrana antes de sumergirla en la solución de ácido fosfórico. Esto es un indicador de la bue- 
na calidad de las membranas sintetizadas.

Como ya se describió, para la caracterización magnética de los arreglos obtenidos, se midieron los ciclos de histéresis a temperatura ambiente, en dos orientaciones relativas entre los ejes longitudinales de los hilos y el campo aplicado: $\theta=0^{\circ}$ (PA: campo magnético paralelo a la dirección del eje longitudinal de los nanohilos) y $\theta=90^{\circ}$ (PE: campo magnético perpendicular a este eje).

El momento magnético total del arreglo posee contribuciones del sustrato de $\mathrm{Al}$ (paramagnético), de la membrana de alúmina (diamagnética) y de los nanohilos de Ni (ferromagnéticos). Para el análisis de las propiedades de histéresis se tuvo en cuenta sólo la contribución ferromagnética y los ciclos de histéresis que se muestran están corregidos, habiendo realizado la sustracción de las contribuciones diamagnéticas y paramagnéticas, ajustadas por las funciones lineales correspondiente. En las Figs. 4 (a)-(d) pueden observarse los resultados para las cuatro muestras estudiadas.
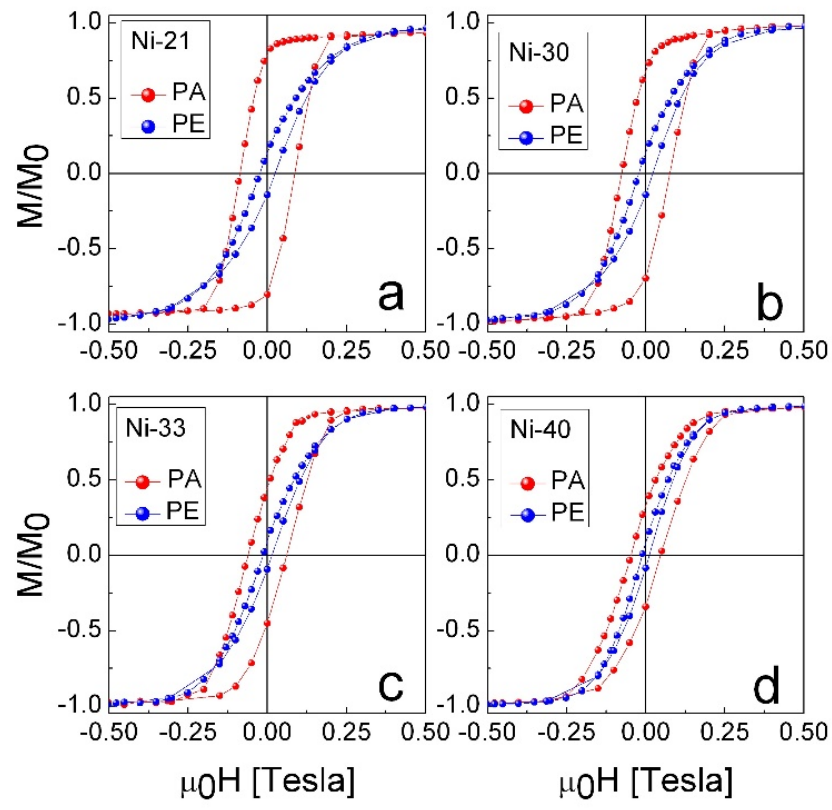

Figura 4: Ciclos de histéresis en configuración PA (círculos rojos) y PE (círculos azules) para las muestras (a) Ni-21; (b) Ni-30; (c) Ni-33 (d) Ni-40. En el eje vertical se grafica la magnetización normalizado por el máximo valor alcanzado por el sistema con un campo aplicado de $2 \mathrm{~T}$.

La diferencia entre los ciclos PA y PE es notable, observándose valores de coercitividad y remanencia mucho mayores para la configuración PA, lo cual indica que la dirección de fácil magnetización de los hilos es a lo largo de su eje longitudinal. Esto se atribuye a una anisotropía efectiva uniaxial de los hilos en la dirección de este eje.

La muestra Ni-21, que tiene la mayor relación de aspecto ( $A r=21 \pm 2$ ), es la que presenta mayor diferencia entre los ciclos PA y PE. A medida que la relación de aspecto $A r$ disminuye, éstos se parecen cada vez más entre sí. Este efecto es esperable, ya que para un valor de la relación de aspecto $A r$ próximo a la unidad la anisotropía de forma desaparece y las direcciones entre el campo aplicado y cualquier eje del hilo (o partícula) son equivalentes, quedando presentes sólo las interacciones dipolares y magnetostáticas. El hecho de que los ciclos en configuración PA y PE medidos para la muestra Ni-40 sean similares corrobora esta interpretación.

Para poder explicar los valores de coercitividad obtenidos en las distintas muestras es necesario considerar la anisotropía magnética efectiva, la cual se puede aproximar por la suma de distintas contribuciones. En el caso de un arreglo de nanohilos, un único hilo no está expuesto solamente al campo externo sino también al campo efectivo generado por los vecinos. Así, para determinar la coercitividad del arreglo deben tenerse en cuenta las anisotropías de forma, magneto-cristalina y magneto-elástica, pero además deben considerarse las interacciones magnetostáticas entre los hilos. El efecto de la porosidad $P$ (o de la relación $d / D$ ) es determinante para definir la magnitud de las interacciones de este tipo.

Los valores límites de la porosidad $P$ representan un hilo aislado $(P=0)$ y una lámina continua $(P=1)$. Para casos intermedios, el valor de este parámetro es crucial al definir la dirección de fácil magnetiza- 
ción; para valores altos de la porosidad $P$, el campo de interacción dipolar favorece la magnetización en una dirección del plano de la membrana (in plane), prevaleciendo a veces sobre el eje de fácil anisotropía de los nanohilos individuales (out of plane) [19]. Las interacciones dipolares entre los hilos pueden modelarse con una aproximación de campo medio como un campo de anisotropía uniaxial efectiva orientado perpendicular al eje de los hilos y proporcional a la porosidad: $H d=-P M_{S}$ (donde $M_{S}$ es la magnetización de saturación) $[\underline{20}, \underline{21}]$. Así, un aumento de la porosidad $P$ puede inducir cambios en el eje de anisotropía, incluso cambiarlo de paralelo a perpendicular a la dirección del eje de los hilos. ENCINAS-OROPESA et al. [20] encuentran que para valores de la porosidad $P$ de $35-38 \%$ este cambio se produce, independientemente del diámetro $d$ de los hilos.

En la Fig. 5 se grafica la dependencia de la coercitividad y remanencia reducida (o cuadratura del ciclo) $M_{R} / M_{S}$ en las configuraciones PA y PE (símbolos llenos y vacíos respectivamente) en función de la relación $d / D$. Como puede observarse, ambas magnitudes disminuyen al aumentar este cociente $d / D$.

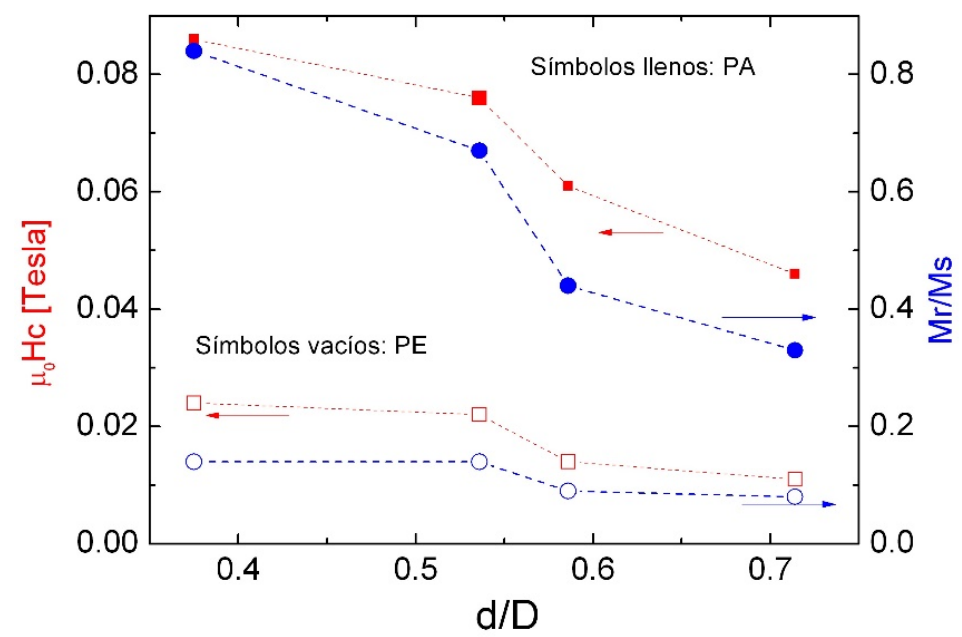

Figura 5: Dependencia de la coercitividad (cuadrados) y remanencia reducida $M r / M s$ (círculos), en configuración PA (símbolos llenos) y PE (símbolos vacíos), en función de la relación $d / D$ para el conjunto de muestras estudiadas.

Para poder interpretar este comportamiento, debe observarse que a medida que el diámetro $d$ aumenta, se produce un acercamiento efectivo entre los nanohilos, ya que la distancia interporo $D$ se mantiene constante. Por ende, las interacciones magnetostáticas comienzan a ser más importantes, por lo que deben tenerse en cuenta. En ese caso, el campo coercitivo estará dado por [22] :

$$
H_{C}=H_{O}-H_{\mathrm{int}}
$$

donde $H_{O}$ es el campo coercitivo correspondiente a un solo hilo y se calcula mediante un modelo adaptado de Stoner-Wholfarth [22] y $H_{\text {int }}$ corresponde al campo disperso inducido en el arreglo de hilos. Expresiones aproximadas para $H_{O}$ y $H_{\text {int }}$ son [22] :

$$
H_{O}=\frac{2 K(w)}{\mu_{0} M_{S}},
$$

donde $w$ es el espesor de la pared de dominio ( $w=52 \mathrm{~nm}$ para Ni [223]); K(w) $=1 / 4 \mu_{0} M_{S}^{2}\left[1-3 N_{z}(w)\right]$ representa la barrera o densidad de energía por unidad de volumen de origen magnetostático que un hilo debe vencer para invertir la magnetización, $N_{z}(w)$ es el factor desmagnetizante que actúa sobre una pared de dominio al nuclear, dado por $N_{z}=1+4 d / 3 \pi w-F_{21}\left[d^{2} / w^{2}\right]$, donde $F_{21}[x]=F_{21}[-1 / 2,1 / 2,2,-x]$ es la función hipergeométrica [24].

$$
H_{\mathrm{int}}=\frac{2 K(L)}{\mu_{0} M_{S}}\left(\frac{\varepsilon E_{\mathrm{int}}(D)}{K(L)}\right)^{2},
$$

donde $\varepsilon$ es un parámetro ajustable que depende de la distribución de los hilos en el espacio (la porosidad $P$ ) y no puede ser calculado a partir de primeros principios, aunque se espera que tome valores entre la unidad y algunas decenas [25]. Por otra parte, $E_{\text {int }}(D)$ es la energía de interacción magnetostática entre dos 
nanohilos separados una distancia $D$, y está dada por [26]:

$$
E_{\text {int }}(D)=2 \mu_{0} M_{S}{ }^{2} \frac{D}{L}\left(\frac{d}{D}\right)^{2}\left(1-\frac{1}{\sqrt{1+(L / D)^{2}}}\right)^{1 / 2}
$$

En la Ec. (5), $\varepsilon E_{\text {int }}(D)$ representa la barrera de energía que debe superar el sistema para invertir su magnetización.

En la Fig. 6 se muestran los valores de coercitividad medidos en configuración PA comparados con los valores calculados usando la Ec. (3), y los valores de $\varepsilon$ elegidos. Puede verse un buen acuerdo entre los resultados teóricos y experimentales. Las desviaciones observadas entre los datos medidos y calculados pueden atribuirse a la dispersión en las longitudes y las posiciones de los nanohilos en los arreglos, además del uso de la expresión dada por la Ec. (6) en los límites de su rango de aplicación, ya que es válida para la condición $A r>>1$ [26]. También debe mencionarse que el modelo teórico no considera la anisotropía magnetocristalina del sistema que podría estar presente, dado el método de síntesis de los nanohilos. Sin embargo, la adición de este tipo de anisotropía no modificaría cualitativamente los resultados obtenidos [22] (su valor debería ser comparable con la anisotropía de forma (del orden de $10^{5} \mathrm{~J} / \mathrm{m}^{3}$ ), pero a temperatura ambiente sólo alcanza los $10^{3} \mathrm{~J} / \mathrm{m}^{3}$ ).

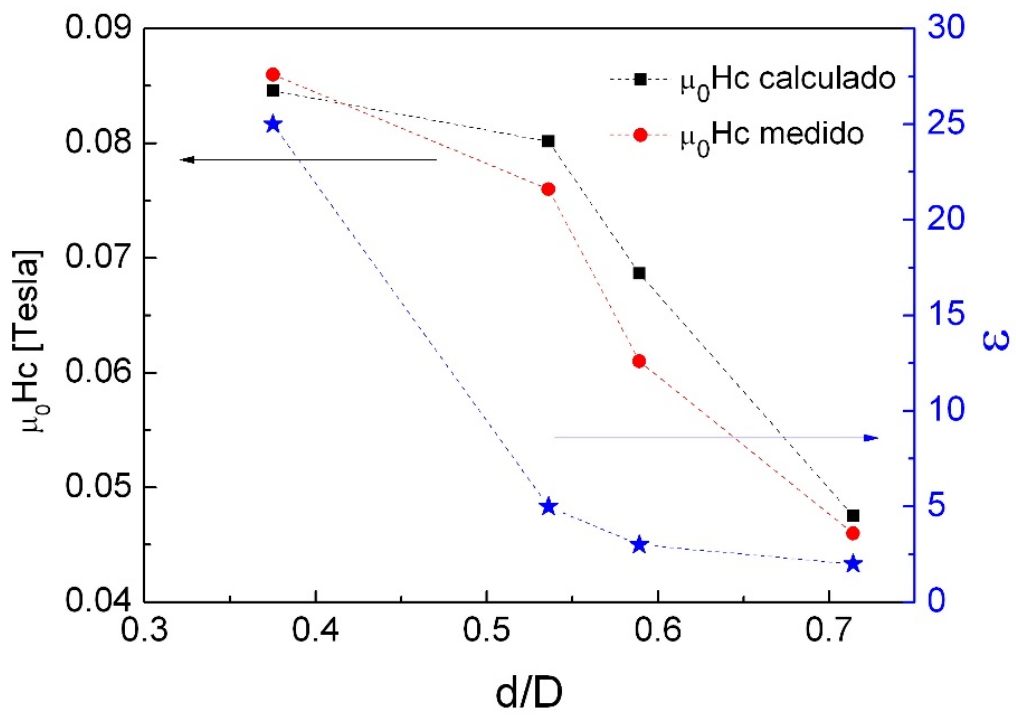

Figura 6: Campo coercitivo medido en configuración PA (cuadrados) y campo coercitivo calculado con la Ec. (3) (círculos) en función de la relación $d / D$. Las estrellas representan los valores del parámetro $\varepsilon$ considerado para cada muestra (referirse al eje de la derecha).

En este trabajo, a diferencia de otros autores que eligen un valor de $\varepsilon$ constante $(\varepsilon=20)$ para arreglos similares de nanohilos de Ni [22], se considera un valor distinto de este parámetro para cada muestra estudiada (ver Fig. 6). Esto es razonable si se tiene en cuenta que al aumentar la porosidad $P$ la barrera de energía disminuye, con la consecuente reducción de coercitividad.

\section{CONCLUSIONES}

Se lograron sintetizar membranas de alúmina porosa autoensambladas con un alto grado de ordenamiento hexagonal. Se variaron los diámetros de los poros por inmersión en ácido fosfórico, manteniendo fija la distancia interporo. Usando estas membranas como moldes, se sintetizaron arreglos de nanohilos de Ni por electrodeposición, que se caracterizaron estructural y magnéticamente. Se encontró que las interacciones magnetostáticas dipolares deben tenerse en cuenta para explicar las reducciones en coercitividad y remanencia observadas en función del aumento de porosidad. El acuerdo entre los resultados experimentales y el modelo teórico evaluado es bueno si se considera la variación del parámetro de ajuste $\varepsilon$ : éste da cuenta de la dependencia de la barrera de energía para la inversión de la magnetización con la porosidad de las muestras. Se encontró que mientras menor es la porosidad del sistema, mayores son las barreras de energía a superar. Una 
interpretación de este comportamiento es que al estar los nanohilos más aislados (porosidad baja) prima su fuerte anisotropía de forma y el sistema se hace magnéticamente más duro. Este aporte es una contribución inédita en el estudio de estos sistemas.

\section{AGRADECIMIENTOS}

Los autores desean agradecer a la Dra. Silvia Urreta por fructíferas discusiones y al LAMARX por el uso del SEM. El trabajo fue realizado con el apoyo de Fundación Holcim, SECyT-UNC y Conicet.

\section{BIBLIOGRAFÍA}

[1] ATKINSON, D., ALLWOOD, A., XIONG, G., et al., "Magnetic domain-wall dynamics in a submicrometre ferromagnetic structure”, Nature Materials, v. 2, n. 2, pp. 85-87, 2003.

[2] THOMAS, L., HAYASHI, M., JIANG, X., et al., "Oscillatory dependence of current-driven magnetic domain wall motion on current pulse length”, Nature, v. 443, n. 7108, pp. 197-200, 2006.

[3] PARKIN, S.S.P., HAYASHI, M., THOMAS, L. “Magnetic Domain-Wall Racetrack Memory”, Science, v. 320, n. 5873, pp. 190-194, 2008.

[4] FERAIN, E., LEGRAS, R. "Industrial applications of ion track technology “, Nucl. Instrum. Methods Phys. Res. B, v. 267, n. 6, pp. 1019-1022, 2009.

[5] GAO, N., WANG, H. J., YANG, E. H. "An experimental study on ferromagnetic nickel nanowires functionalized with antibodies for cell separation”, Nanotechnology, v. 21, n. 10, pp. 105107, 2010.

[6] SCHELHAS, L. T., BANHOLZER, M. J., MIRKIN, C. A., et al., "Magnetic confinement and coupling in narrow-diameter Au-Ni nanowires”, Mag. Magn. Mater., v. 379, n. 1, pp. 239-243, 2015.

[7] HAMIDI, S. M., SOBHANI, A., AFTABI, A., "Optical and magneto-optical properties of aligned Ni nanowires embedded in polydimethylsiloxane”, J. Mag. Magn. Mater., v. 374, n. 1, pp. 139-143, 2015.

[8] YALÇIN, O., KARTOPU, G., ÇETIN, H., et al, "A comparison of the magnetic properties of Ni and Co nanowires deposited in different templates and on different substrates", J. Mag. Magn. Mater., v. 373, n. 1, pp. 207-212, 2014.

[9] LAVÍN, R., DENARDIN, J. C., ESCRIG, J., et al., “Angular dependence of magnetic properties in Ni nanowire arrays “, J. Appl. Phys., v. 106, n. 10, pp. 103903, 2009.

[10] ZENG, H., MICHALSKI, S., KIRBY, R. D., "Effects of surface morphology on magnetic properties of Ni nanowire arrays in self-ordered porous alumina”, J. Phys.: Condens. Matter, v. 14 n. 4, pp. 715, 2009.

[11] CARIGNAN, L., MASSICOTTE, M., CALOZ, C., "Magnetization Reversal in Arrays of Ni Nanowires With Different Diameters”, et al., IEEE Trans. Mag., v. 45, n. 10, pp. 4070-4073, 2009.

[12] TIAN, F., HUANG, Z. P., WHITMORE, L. "Fabrication and magnetic properties of Ni nanowire arrays with ultrahigh axial squareness”, Phys. Chem. Chem. Phys., v. 14, pp. 8537, 2012.

[13] Q-F ZHAN, J-H GAO, Y-Q LIANG, et al., "Dipolar interactions in arrays of iron nanowires studied by Mössbauer spectroscopy”, Phys. Rev. B, v. 72, n. 2, pp. 024428, 2005.

[14] NIELSCH, K., WEHRSPOHN, R. B., BARTHEL, J., "High density hexagonal nickel nanowire array", et al., J. Mag. Magn. Mater., v. 249, pp. 234-240, 2002.

[15] CULLITY, B., GRAHAM, C. Introduction to Magnetic Materials, 2 ed., Wiley, New Jersey, 2009.

[16] THOMPSON, G. E. "Porous anodic alumina: fabrication, characterization and applications",Thin Solid Films, v. 297, n. 1, pp. 192-201, 1997.

[17] YIN, K.-M., LIN, B.-T. "Effects of boric acid on the electrodeposition of iron, nickel and iron-nickel”, Surface and Coatings Technology, v. 78, pp. 205-210, 1996.

[18] NIELSCH, K., CHOI, J., SCHWIRN, K., et al., "Self-Ordering Regimes of Porous Alumina: The $10 \%$ Porosity Rule”, Nano Lett., v. 2, n. 7, pp. 677-680, 2002.

[19] DMYTRIIEV, O., AL-JARAH, U. A. S., GANGMEI, P., "Static and dynamic magnetic properties of densely packed magnetic nanowire arrays”, Phys. Rev. B, v. 87, n. 17, pp. 174429, 2013.

[20] ENCINAS-OROPESA, A., DEMAND, M., PIRAUX, L. "Dipolar interactions in arrays of nickel nanowires studied by ferromagnetic resonance”, Phys. Rev. B, v. 63, n. 10, pp. 104415, 2001.

[21] MAURER, T., ZIGHEM, F., FANG, W., et al., "Influence of the dipolar interactions in magnetic nanowires aggregates”, J. Appl. Phys., v. 110, pp. 123924, 2011. 
[22] ESCRIG, J., LAVÍN, R., PALMA, J. L., et al., “Geometry dependence of coercivity in Ni nanowire arrays”, Nanotechnology, v. 19, n. 7, pp. 075713, 2008.

[23] BERTOTTI, G. Hysteresis in Magnetism, Academic Press Inc. San Diego, California, 1998.

[24] BELAGGIA, M., TANDON, S., ZHU, Y., et al., "On the magnetostatic interactions between nanoparticles of arbitrary shape”, Mag. Magn. Mater., v. 278, n. 1, pp. 270-284, 2004.

[25] ALLIA, P., COISSON, M., TIBERTO, P., “Granular Cu-Co alloys as interacting superparamagnetset al”., Phys. Rev. B, v. 64, n. 14, pp. 144420, 2001.

[26] VÁZQUEZ, M., PIROTA, K., HERNÁNDEZ-VÉLEZ, M., “Magnetic properties of densely packed arrays of Ni nanowires as a function of their diameter and lattice parameter”, J. Appl. Phys., v. 95, n. 11, pp. 6642, 2004. 